\title{
Der Heilige Geist und die Realisierung des Glaubens in der Geschichte. Überlegungen zur systematischen Funktion der Pneumatologie
}

\begin{tabular}{|c|c|}
\hline \multicolumn{2}{|c|}{$\begin{array}{l}\text { Authors: } \\
\text { Christian Danz }{ }^{1,2}\end{array}$} \\
\hline \multicolumn{2}{|c|}{$\begin{array}{l}\text { Affiliations: } \\
{ }^{1} \text { Systematische Theologie, } \\
\text { Evangelisch-Theologischen } \\
\text { Fakultät, Universität Wien, } \\
\text { Austria }\end{array}$} \\
\hline \multicolumn{2}{|c|}{$\begin{array}{l}{ }^{2} \text { Department of Dogmatics } \\
\text { and Christian Ethics, University } \\
\text { of Pretoria, South Africa }\end{array}$} \\
\hline \multicolumn{2}{|c|}{$\begin{array}{l}\text { Project leader: J. Buitendag } \\
\text { Project number: } 02402343\end{array}$} \\
\hline \multicolumn{2}{|c|}{$\begin{array}{l}\text { Prof. Dr Christian Danz is part } \\
\text { of the research project, } \\
\text { 'Theology and Nature', } \\
\text { directed by Prof. Dr Johan } \\
\text { Buitendag, Department of } \\
\text { Dogmatics and Christian Ethics, } \\
\text { Faculty of Theology, University } \\
\text { of Pretoria and Dean of the } \\
\text { Faculty of Theology. }\end{array}$} \\
\hline \multicolumn{2}{|c|}{$\begin{array}{l}\text { Corresponding author: } \\
\text { Christian Danz, } \\
\text { Christian.danz@univie.ac.at }\end{array}$} \\
\hline $\begin{array}{l}\text { Dates: } \\
\text { Received: } 17 \text { [ } \\
\text { Accepted: } 31 \\
\text { Published: } 29\end{array}$ & $\begin{array}{l}\text { ec. } 2015 \\
\text { an. } 2016 \\
\text { July } 2016\end{array}$ \\
\hline \multicolumn{2}{|c|}{$\begin{array}{l}\text { How to cite this article: } \\
\text { Danz C., 2016, 'Der Heilige } \\
\text { Geist und die Realisierung } \\
\text { des Glaubens in der } \\
\text { Geschichte. Überlegungen } \\
\text { zur systematischen Funktion } \\
\text { der Pneumatologie', HTS } \\
\text { Teologiese Studies/ } \\
\text { Theological Studies 72(4), } \\
\text { a3293. http://dx.doi. } \\
\text { org/10.4102/hts.v72i4.3293 }\end{array}$} \\
\hline \multicolumn{2}{|c|}{$\begin{array}{l}\text { Copyright: } \\
\text { (C) 2016. The Authors. } \\
\text { Licensee: AOSIS. This work } \\
\text { is licensed under the } \\
\text { Creative Commons } \\
\text { Attribution License. }\end{array}$} \\
\hline \multicolumn{2}{|l|}{ Read online: } \\
\hline 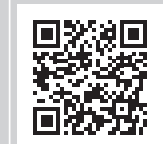 & $\begin{array}{l}\text { Scan this QR } \\
\text { code with your } \\
\text { smart phone or } \\
\text { mobile device } \\
\text { to read online. }\end{array}$ \\
\hline
\end{tabular}

Die systematische Funktion der Lehre vom Heiligen Geist für die theologische Dogmatik ist umstritten. Der Beitrag arbeitet in einer problemgeschichtlichen Perspektive die These aus, dass der Pneumatologie eine notwendige und eigene Funktion für die Explikation der Religion zukommt. Deren Thema ist, wie zu zeigen sein wird, das Wissen des religiösen Aktes um seine Einbindung in eine inhaltliche Überlieferung sowie deren Wandelbarkeit.

\section{Einführung}

In seiner Studie zur protestantischen Pneumatologie im 20. Jahrhundert Die evangelische Lehre vom Heiligen Geist und seiner Person kommt Christian Henning (2000) zu dem Resultat einer heillosen Konfusion über das,

$[W]$ as man unter dem (Heiligen) Geist und seinem Wirken verstehen soll. Ersichtlich ist, daß jeder Theologe mit seinem individuellen Beitrag zum pneumatologischen Diskurs zur Entstehung und Vergrößerung dieser Konfusion beigetragen hat. [...] So entstand aus der allen Pneumatologien anhaftenden Mehrdeutigkeit des Geistverständnisses schließlich unübersichtliche Vieldeutigkeit. (S. 297) ${ }^{1}$

Die von Henning diagnostizierte Konfusion über den Gottesgeist hat ihren Grund nicht allein in der Unschärfe des Geistbegriffs, sondern auch in der Lehrentwicklung sowie der daraus resultierenden unklaren Stellung der Pneumatologie im Aufbau der theologischen Dogmatik. Erörtert wird der Gottesgeist in der Trinitätslehre, der Lehre von der Heilszueignung (Soteriologie) und der von der Ekklesiologie, aber auch in der Schriftlehre sowie der Eschatologie und der Lehre von der Schöpfung. Als eine eigenständige dogmatische Lehre ist die Pneumatologie nicht ausgearbeitet worden, sie wurde ausschließlich in Verbindung mit anderen dogmatischen Lehrstücken abgehandelt. ${ }^{2}$ Freilich verbindet schon der dritte Artikel des Glaubensbekenntnisses sehr unterschiedliche Themen im Zusammenhang mit dem Glauben an den Heiligen Geist. Die hier mit diesem aufgeführten Aspekte: die Kirche, die Gemeinschaft der Heiligen, die Vergebung der Sünden, die Auferstehung der Toten sowie das ewige Leben, scheinen nicht viel miteinander zu tun zu haben und im Vergleich mit den ersten beiden Artikeln: Gott der Schöpfer und Jesus der Christus, eher lose zusammengefügt zu sein. All das hatte zur Folge, dass schon in der klassischen Dogmatik des Protestantismus die Zuordnungen des Geistes äußerst vielschichtig ausgefallen sind. ${ }^{3}$ Worin seine spezifische dogmatische Funktion besteht ist alles andere als deutlich.

Vor dem skizzierten Hintergrund verwundert es nicht, wenn entweder genau diese Unfassbarkeit und Vieldeutigkeit als das Eigentümliche des Gottesgeistes erklärt (Welker 2013) oder gar vorgeschlagen wird, die Pneumatologie als eigenständige dogmatische Lehre aufzugeben. 'Es fragt sich jedoch', so Dietrich Ritschl (1993):

$[\mathrm{Ob}]^{\prime}$ der Geist Gottes (des Vaters und des Sohnes) wie etwas Eigenständiges thematisiert, Greifbares definiert und wie etwas Verständliches expliziert werden kann. Er steht ja - so könnte eingewandt werden - Gott (dem Vater, dem Schöpfer) und Jesus (in dem Gott gegenwärtig war) nicht wie etwas Separates gegenüber, auch nicht den Gläubigen, denn deren Glaube wird ja gerade ein Werk des hl. Geistes genannt. [...] So scheint der Rufnach einer neuen, verständlicheren P[neumatologie] ein unerfüllbarer Wunsch zu sein. (S. 1247)

1.Vergleiche hierzu den Überblick über die neuere Debatte bei Wittekind (2014:62-66).

2.So schon das Urteil von Trillhaas (1962:405): 'Die Lehre vom Heiligen Geist hat die Eigentümlichkeit, daß sie in der Geschichte der kirchlichen Lehrbildung eigentlich nur in bestimmten Kombinationen erscheint.'

3.So das Urteil von Wittekind (2014:14). 
Da der Heilige Geist lediglich eine Objektivation des Glaubens darstellt, könne ihm keine Eigenständigkeit zukommen.

Das Verständnis des Heiligen Geistes, die angedeuteten Aspekte unterstreichen das, ist in der protestantischen Dogmatik nicht erkenntlich. ${ }^{4}$ Um hier Klarheit zu schaffen, ist es notwendig, die systematische Funktion der Pneumatologie für die Dogmatik zu analysieren. Diese unterliegt ebenso wie die Lehre vom Gottesgeist seit der Aufklärung einer tiefgreifenden Umformung. Die moderne Entwicklungsgeschichte der theologischen Dogmatik führte, davon wird in den folgenden Überlegungen ausgegangen, zu einer Rekonstruktion des Religionsbegriffs in der Theologie. ${ }^{5}$ Um also die systematische Funktion des Geistes $\mathrm{zu}$ bestimmen, ist der Religionsbegriff in den Blick zu nehmen. Erst vor diesem Hintergrund wird es möglich, die spezifische Funktion des Gottesgeistes für die Dogmatik zu benennen.

Damit ist das Thema der nachfolgenden Überlegungen zur dogmatischen Funktion der Pneumatologie umrissen. Einzusetzen ist mit einer knappen Problemgeschichte des pneumatologischen Lehrstücks in der Moderne. Im zweiten Abschnitt ist der Religionsbegriff in den Blick zu nehmen. Abschließen werde ich mit ein paar Überlegungen zur systematischen Funktion der dogmatischen Geistlehre. Diese besteht, so die zu entfaltende These, in dem Wissen des religiösen Aktes um seine Einbindung in eine konkrete, inhaltlich bestimmte religiöse Tradition und deren Wandelbarkeit.

\section{Die Funktion des Gottesgeistes in der protestantischen Dogmatik}

Martin Luther (1982) hat die spezifische Funktion des Geistes in seiner Erklärung des dritten Artikels des Kleinen Katechismus soteriologisch bestimmt.

Ich gläube, daß ich nicht aus eigener Vernunft noch Kraft an Jesum Christum, meinen Herrn gläuben oder zu ihm kommen kann, sondern der heilige Geist hat mich durchs Evangelium berufen, mit seinen Gaben erleuchtet, im rechten Glauben geheiligt und erhalten. (S. 511f.)

Der Wittenberger Reformator bindet den Gottesgeist an Christus und versteht jenen als Vermittlung von diesem in der Geschichte. Luther konstruiert die Pneumatologie allerdings nicht nur als geschichtliche Übermittlung Christi, wichtiger ist ein weiterer Aspekt. ${ }^{6}$ Er unterscheidet beim Gottesgeist ähnlich wie bei der Schrift zwischen einer äußeren und einer inneren Dimension und bindet letztere an erstere zurück. Die Schrift sei, wie Luther gegen die sogenannten Schwärmer und die römische Kirche geltend

4.Vergleiche hierzu auch Danz \& Murrmann-Kahl (2014); Düsing, Neuer \& Klein (2009).

5.Vergleiche hierzu Danz (2010)

6.Zu Luthers Pneumatologie vergleiche. Wittekind (2014:16-20). Zu Luthers Theologie vergleiche Danz (2013). gemacht hat, das Vehikel des Geistes. ${ }^{7}$ Dieser bedient sich bei seinem inneren heilschaffenden Wirken des äußeren Schriftwortes. Unabhängig und losgelöst vom biblischen Buchstaben gibt es keine Erfahrungen des Heiligen Geistes. Bestenfalls seinen Phantasien und dem eigenen Geist begegne man dann. Der aber führt nicht zur Gewissheit des Heils. Diese resultiert jedoch noch nicht aus dem äußeren Wirken des Geistes, sie verdankt sich seinem inneren Wirken. Der Gottesgeist beschreibt in der Theologie des Reformators die individuelle Aneignung Christi, allerdings so, dass diese keine Handlung des Einzelnen ist, sondern Gnade Gottes. Die Pneumatologie stellt damit die notwendige Aneignung des Glaubens (das Bild Christi) als einen Akt dar, der kein menschlicher Vollzug ist. Sie thematisiert die Struktur der religiösen Gewissheit selbst, an äußerliche Medien gebunden, aber aus diesen weder ableitbar noch vom Menschen selbst herstellbar zu sein.

Die reformatorische Deutung des Gottesgeistes wurde von der Theologie des 19. Jahrhunderts aufgenommen und vor dem Hintergrund der historischen und erkenntnistheoretischen Kritik an den biblischen und metaphysischen Grundlagen der altprotestantischen Theologie die Pneumatologie gleichsam als Beschreibung der Realisierung der Religion in der Geschichte durchgeführt: So in Schleiermachers pneumatologischer Konstruktion der Ekklesiologie (vgl. Schleiermacher [1830-1831] 1999:§§ 121-125) oder in Albrecht Ritschls Lehre vom Heiligen Geist. Die Pneumatologie des Göttinger Theologen beschreibt die Verwirklichung des Reiches Gottes in der Geschichte als Neubestimmung des Menschen im Glauben - als Herrschaft über die Welt, gegen diese - in seiner Ausrichtung auf den Endzweck. 'Die Uebereinstimmung dieser Antriebe mit dem Zwecke Gottes und die Gleichheit derselben in allen Einzelnen wird begründet und verbürgt durch den heiligen Geist in der Gemeinde' (Ritschl 1890:42f. [§ 46]). Der Heilige Geist steht für die allen Mitgliedern der Gemeinde gemeinsame Bestimmung, also die Allgemeinheit der sittlichen Bestimmung zum Reich Gottes. Sie deckt sich mit der 'Selbsterkenntniß Gottes' (Ritschl 1890:42f. [§ 46]), nämlich der reflexiven Durchsichtigkeit (dem Glauben) als Endzweck der Schöpfung. Indem die Mitglieder der Gemeinde im individuellen Vollzug des Glaubens die Selbsterkenntnis Gottes realisieren, überwinden sie ihre geschichtliche Besonderheit, so dass das Allgemeine des sittlichen Endzwecks der alleinige Bestimmungsgrund ihres Handelns ist. Die Überwindung der Welt gegen die Welt (die geschichtliche Besonderheit und die natürlichen Ordnungen)

7.Vergleiche Luther, Wider die himmlischen Propheten, WA 18; 136. 139. Žtiert nach Hirsch (1937:76f.): 'So nu Gott sein heiliges Euangelion hat aus lassen gehen, handelt er mit uns auf zweierlei Weise. Einmal äußerlich, das ander Mal innerlich. Äußerlich handelt er mit uns durchs mündliche Wort des Euangelii und durch leibliche Zeichen, als do ist Taufe und Sakrament. Innerlich handelt er mit uns durch den heiligen Geist und Glauben samt andern Gaben. Aber das alles der Maßen und der Ordnung, daß die äußerlichen Stücke sollen und müssen vorhergehen und die innerlichen hernach und durch die äußerlichen kommen, also daß ers beschlossen hat, keinem Menschen die innerlichen Stück zu geben ohn durch die äußerlichen hat, keinem Menschen die innerlichen Stück zu geben ohn durch die außerlichen Stücke. Denn er will niemand den Geist noch Glauben geben ohn das außerliche Wort und Zeichen, so er dazu eingesetzt hat. [ ] Zuerst vor allen Werken und Dingen höret man das Wort Gottes, darin der Geist die Welt um die Sünde straft, Joh. 16 (8). Wenn die Sünde erkennet ist, höret man von der Gnade Christi, im selben Wor kommt der Geist und gibt den Glauben, wo und welchem er will. Darnach geht an die Tötung und das Kreuz und die Werk der Liebe. Wer dir eine andere Ordnung vorschlägt, da zweifel nicht, es sei der Teufel. [ ].' 
wird zum Antrieb ihres Handelns. ${ }^{8}$ Dafür steht bei Ritschl der Heilige Geist. ${ }^{9}$ Er expliziert die reflexive Struktur des Glaubensaktes in seiner geschichtlichen Realisierung.

Ähnlich wie Ritschl konstruiert der Hallenser Theologie Martin Kähler die Pneumatologie in seinem systematischen Hauptwerk Die Wissenschaft der christlichen Lehre als Beschreibung der Weise, wie sich die Religion in der Geschichte realisiert.

Das Handeln Gottes, in welchem er die Versöhnung zueignet, vollzieht sich durch den heiligen Geist. Das entsprechende Wirken des Geistes wird in seiner Art durch die Veränderung bedingt, welche die Versöhnung der Welt in das Verhältnis der Menschheit zu Gott gebracht hat, und das findet seinen Ausdruck in dem Bekenntnisse zu dem Geist, den der Vater als den Geist seines Sohnes ausgegossen hat oder durch den er sich in dem persönlichen Leben der Menschheit stetige wirksame Gegenwart gibt, und zwar ebenso geschichtlich als in den einzelnen Glaubenden (Kähler [1905] 1994:381).

Der Heilige Geist beschreibt die Zueignung der Versöhnung an den Einzelnen, die stets in die Geschichte eingebunden ist. Dementsprechend behandelt Kähler den Geist in der Lehre von der Kirche, die im Unterschied zum traditionellen Aufbau der lutherischen Dogmatik vor der Soteriologie abgehandelt wird. Die Funktion der Pneumatologie ist es, die reflexive Struktur des rechtfertigungstheologisch gedeuteten Glaubensaktes sowie dessen geschichtliche Vermittlung zu reflektieren. Der Gottesgeist repräsentiert das Wissen des in die geschichtliche Vermittlung der Versöhnung eingebundenen Glaubensaktes um seine eigene Unableitbarkeit aus dieser. Spekulative Erwägungen über das Wesen Gottes und seine trinitarische Verfasstheit treten demgegenüber in den Hintergrund. ${ }^{10}$

Dem folgt in der ersten Hälfte des 20. Jahrhunderts auch noch Karl Barth. In seinem [1930] publizierten Beitrag Der heilige Geist und das christliche Leben bestimmt er den Gottesgeist als unableitbares Ereignis des Glaubens:

1. Der heilige Geist im Ereignis seines Daseins für den Menschen ist die alleinige Wirklichkeit von dessen Gottebenbildlichkeit. Diese ist also nicht und wird nicht eine Eigenschaft des geschaffenen Geistes, sondern sie ist und bleibt das freie nur als Gnade begreifliche, vom Menschen aus immer unbegreifliche Werk des Schöpfers an seinem Geschöpf. 2. Christliches Leben ist das durch den heiligen Geist für das Wort geöffnete Menschenleben.

8.Vergleiche Ritschl (1890:42f [S 46]): 'Aus diesen Rücksichten ist der Gemeingeist, in welchen die Glieder der Gemeinde ihre gleiche Gotteserkenntniß und ihre gleichen Antriebe zum Reich Gottes und zur Gotteskindschaft gewinnen, der heilige Geist. Vergleiche auch Ritschl (1999:56): 'Das Allgemeine [sc. der Endzweck des Reiches Gottes als Aufgabe der Gemeinde] ist immer nur im Besonderen wirklich [sc. als individueller Vollzug in der Geschichte und ihren sittlichen Ordnungen]; jede Naturgattung besteht in ihren Arten. Die christlichen Regeln des sittlichen Handeln [sc. die Bestimmung des Menschen zum allgemeinen sittlichen Endzweck de Reiches Gottes] also ist die, daß man alle besonderen Verbindungen des natürlichen Wohlwollens [sc. Ehe, Familie, Staat etc.] durch die allgemeine Fertigkeit der Liebe zu den Menschen als solchen veredle, und über die Natur zur Regel des Geistes erhebe.'

9.Vergleiche hierzu Danz (2015a:257-272).

10.Vergleiche hierzu Wittekind (2014:38): 'In vielen protestantischen Dogmatiken [sc. am Ende des 19. Jahrhunderts] steht die Betonung der Geschichtlichkeit des Geistes, und zwar sowohl in kirchlicher als auch in individueller Hinsicht, für die Abkehr von magisch oder übernatürlich (oder katholisch) genannten Vorstellungen von der Kraft des Geistes.'
Der heilige Geist bedeutet also in seinem Dasein und Wirken die subjektive Seite im Ereignis der Offenbarung. (S. 39)

Der Heilige Geist wird von Barth dynamisiert und als Ereignis der Offenbarung Gottes verstanden. Der Sinn dieser Fassung der Pneumatologie besteht in der Ablösung des Gottesgeistes von anthropologischen Voraussetzungen. Der göttliche Geist ist weder eine Eigenschaft noch eine religiöse Anlage im Menschen. Er ist allein als unableitbarer Vollzug der Gotteserkenntnis wirklich. Das ist der systematische Gehalt der Bindung des Geistes an die Offenbarung Gottes. Barth beschreibt mit dem Heiligen Geist die Selbstverwirklichung der wahren Religion (Glaube), die nur als und im Vollzug wirklich ist.

Die Theologie der zweiten Hälfte des 20. Jahrhunderts hat eine solche Fassung der Pneumatologie als Engführung kritisiert. In der Tat, die enge Verknüpfung von Geist und Schrift blockierte die Aufmerksamkeit auf die religiöse Entwicklung in der modernen Gesellschaft. Schon Wolfgang Trillhaas hatte in seiner Dogmatik, gegen die von den Reformatoren geltend gemachte Bindung des Geistes an das Wort der Schrift, für eine Erweiterung der Geistlehre und eine Einbeziehung des Enthusiasmus und Spiritualismus plädiert (vgl. Trillhaas 1962:421-426; Tillich 1966:137-144). Andernfalls würde die protestantische Theologie sowohl der 'unsichtbaren Religion' der Moderne (Thomas Luckmann) als auch den geistinspirierten und ekstatischen Neuaufbrüchen in vielen protestantischen Kirchen verständnislos gegenüberstehen. Dem versuchte man nun durch eine Ausweitung des Geistbegriffs Rechnung zu tragen. Der Heilige Geist sollte von seiner vormaligen, als zu eng empfundenen Bindung an das Schriftwort und seine soteriologische Funktion gelöst werden. Der Gottesgeist, so Jürgen Moltmann schon in seinem Vortrag Die Wahrnehmung der Geschichte in der christlichen Sozialethik aus dem Jahre 1960, manifestiere sich in den schöpferischen Aufbrüchen der 'Verhärtung der Geschichte menschlicher Gesellschaften'.11 In seiner späteren Pneumatologie Der Geist des Lebens hat der Tübinger Theologe diesen Gedanken weiter ausgebaut und den Gottesgeist in den Erfahrungen eschatologischer Ganzheit des Lebens sowie der Befreiung von Leid, Unterdrückung und der Stiftung von Hoffnung identifiziert (Moltmann 1991). Dass der Geist Gottes nicht nur in der Kirche wirkt, sondern in den polyphonen Erfahrungen von Befreiung sowie der Bewältigung weltweiter Krisen, ist auch die Überzeugung des Moltmann-Schülers Michael Welker. In seiner realistischen Pneumatologie, die zuerst 1992 unter dem Titel Gottes Geist. Theologie des Heiligen Geistes erschien, wird gerade die Unfassbarkeit und unberechenbare Vielfältigkeit zum Wesen des Gottesgeistes erklärt.

Der Geist gibt die schöpferische Macht Gottes zu erkennen, die die Vielfalt des Geschöpflichen in reiche, fruchtbare, lebenserhaltende, stärkende und schützende Beziehungen bringt.

11.Vergleiche Moltmann (1968:156): 'Immer wird die Verhärtung der Geschichte menschlicher Gesellschaften in einem die Zukunft aufhebenden status gesprengt menschlicher Gesellschaften in einem die Zukunft authebenden status gesprengt
werden von der eschatologischen Unabgeschlossenheit des Menschen, die sich werden von der eschatologischen Unabgeschlossenheit des Menschen, die sich
zeigt in der Langeweile oder in den Revolutionen, zu denen der Geist durch alle Versuche, die Geschichte aufzuheben, provoziert wird.' 
Der Geist Gottes offenbart die Kraft des Erbarmens Gottes, die Gott besonders den Schwachen, Vernachlässigten, Ausgegrenzten und Hinfälligen zuwendet. (Welker 2013:16). ${ }^{12}$

Eine Ausweitung des Gottesgeistes, verbunden mit seiner Ablösung von seiner traditionellen soteriologischen Bindung an die Schrift sowie an Christus, wird auch in neueren Konzeptionen einer pluralismusoffenen Theologie der Religionen betrieben. So hat der in Basel lehrende Systematische Theologe Reinhold Bernhardt in einer Vielzahl von Publikationen für eine pneumatologische Grundlegung einer Religionstheologie plädiert (2005b; vgl. Bernhardt 2000:287-301; 2005a; 2005b:107-120).

Der trinitätstheologische Ansatz verweist [...] auf die allgegenwärtige Geisteskraft Gottes, die den gesamten kosmischen Prozeß durchdringt und im Menschen als Inspiration wirkt, die ihn zur Erkenntnis des Seinsgrundes führt. [...] Die religiösen Traditionen können und müssen aus dieser Perspektive als Ereignisräume der wirksamen Gegenwart Gottes angesehen werden (S. 118f., vgl. Bernhardt 2005b: 119; vgl. Bernhardt 2005a:243).

Gegen die pluralistische Religionstheologie und ihre Voraussetzung eines allen Religionen zugrundeliegenden Absoluten wird hier aus der Perspektive der christlichen Religion der Gottesgeist als Bezugspunkt gesetzt. Dadurch soll das Anliegen der pluralistischen Theologie, zu einer positiven Würdigung nichtchristlicher Religionen $\mathrm{zu}$ gelangen, auf eine angemessenere Weise in Angriff genommen werden. Das Postulat des Wirkens des Gottesgeistes in der Welt der Religionen führt allerdings zu einem Verlust von dessen Konturen.

Der Heilige Geist wurde in der Theologie der zweiten Hälfte des 20. Jahrhunderts zur theologischen Beschreibung von Krisenbewältigungsprogrammen, zur Kritik an dem Geist der Welt oder der neuzeitlichen Subjektivität und ihrer Moral sowie schließlich der akademisch betriebenen Theologie selbst. ${ }^{13}$ Seien es die Bewältigung der ökologischen Krise der Weltgesellschaft, politische Programme der Befreiung der Unterdrückten und Marginalisierten, die Bewältigung des Leidens oder die Kreativität des Lebens, all das konnte nun mit dem Gottesgeist beschrieben werden. Die Andersheit des Heiligen Geistes erblickt man jetzt gerade darin, dass seine Wirklichkeit mehr und anders ist als die des Geistes der Welt. Wenn allerdings überall der Geist am Wirken ist, dann verliert er sein spezifisches Profil und wird unbestimmt. Die eingangs genannte Einschätzung von Henning, die Entwicklung der protestantischen Pneumatologie im 20. Jahrhundert komme einer Verfallsgeschichte gleich, hat hieran ihren Anhalt. ${ }^{14}$

12.Ene semiotische Deutung des Heiligen Geistes hat Deuser (1999:152-157) vorgeschlagen. Der Gottesgeist steht hier für die Erschließung der Kreativität de kosmologischen Zeichenprozesses. 'Die Lehre vom Heiligen Geist, die Pneumatologie, ist folglich der Ort, an dem die Einsichten der kategorialen Semiotik [] nicht nur zu wiederholen, sondern zu bestätigen sind. Jetzt erst ist die Semiotik [ ] nicht nur zu wiederholen, sondern zu bestätigen sind. Jetzt erst ist die vollständig ins Bild gekommen' (Deuser 1999:154).

13.Vergleiche hierzu Wittekind (2014:13-67).

14. Henning selbst möchte der Pneumatologie durch eine Neubestimmung der Personalität des Gottesgeistes genauere Konturen verschaffen. Sein Vorschlag, die

\section{Religion als geschichtlich gewordenes menschliches Selbstverständnis}

Um die systematische Funktion der Pneumatologie für die theologische Dogmatik zu rekonstruieren, ist es notwendig, von einem Begriff der Religion auszugehen. Es ist freilich an dieser Stelle nicht möglich, die komplexen Diskurse über den Religionsbegriff seit der Aufklärung hier im Detail nachzuzeichnen (vgl. Danz 2008; Riesebrodt 2007; Barth 2003; Wagner 1991). Wir müssen uns auf einige entscheidende Gesichtspunkte beschränken. Zunächst führten die theologischen, religionsphilosophischen und religionswissenschaftlichen Debatten im 20. Jahrhundert zu einer Auflösung eines anthropologischen Religionsbegriffs, wie er von der Aufklärungstheologie sowie der des 19. Jahrhunderts ausgearbeitet wurde (Danz 2015b:157-185). Religion fußt weder auf einer Anlage im Menschen noch ist sie eine universale anthropologische Konstante. Sie entsteht vielmehr unableitbar im Leben eines Menschen als ein solcher Selbstdeutungsakt, der selbst bereits in eine bestimmte und geschichtlich gewordene Religionskultur eingebunden ist. Damit ist der zweite hier zu nennende Aspekt schon erwähnt, die Bindung der Religion an ihren Vollzug. Unabhängig von einem religiösen Selbstverständnis, welches sich selbst als religiös bezeichnet, kann, wie die Kontroversen über die analytische Prägnanz von funktionalen Religionsbegriffen deutlich gemacht haben, Religion nicht angemessen erfasst werden (Danz 2008:110-112; Riesebrodt 2007:17-42). Und schließlich führte drittens die theologiegeschichtliche Entwicklung im 20. Jahrhundert zu einer Rekonstruktion des Religionsbegriffs in der Theologie. Das ist der Sinn der theologischen Religionskritik, wie sie von der Dialektischen Theologie, aber auch von anderen Theologen wie Paul Tillich, betrieben wurde. Die theologische Beschreibung der wahren Religion als Offenbarung Gottes zielt auf ein gegenüber anthropologischen Religionsbegriffen, die von einer religiösen Anlage im Menschen (religiöses Apriori) ausgehen, angemesseneres Verständnis. Damit wird jedoch die theologische Dogmatik selbst $\mathrm{zu}$ einer symbolischen Darstellung des religiösen Aktes und seiner reflexiven Struktur.

Vor dem skizzierten problemgeschichtlichen Hintergrund kommt Religion als eine geschichtlich gewordene Weise menschlichen Selbstverständnisses in den Blick. Religion ist weder etwas objektiv Vorliegendes noch hat sie eine anthropologische oder gesellschaftliche Funktion. Beide Auffassungen werden ihr nicht gerecht, da sie die Teilnehmerperspektive aus dem Begriff der Religion ausschließen. Vielmehr ist Religion allein im und als das Geschehen des Sich-Verstehens menschlichen Lebens wirklich, indem sie sich selbst als solche darstellt und bezeichnet. Anthropologische und phänomenologische zu verbinden, um auf diese Weise die Personalität des Geistes zu begründen, fungiert
zugleichals Maßstab, an dem diepneumatologischen Konzeptionen derprotestantischen zugleich als Maßstab, an dem die pneumatologischen Konzeptionen
Theologie gemessen werden. Vergleiche Henning (2000:304-431).
} 
Religionsbegriffe sind folglich durch ein Verständnis von Religion als Kommunikation zu ersetzen. ${ }^{15}$ Religion entsteht in ihrem Vollzug sowie in seinem reflexiven Wissen um sich und ist an diesen gebunden. Aufgrund ihrer Vollzugsgebundenheit liegen weder deren Subjekt noch deren Gehalte als dem religiösen Akt bereits vorgegebene fixe Größen vor. Beides sind Bestimmungen des religiösen Aktes (Wittekind 2008:373-384). Das religiöse Selbst entsteht erst in dem selbstbezüglichen Reflexionsakt und stellt seine Selbsterschlossenheit als seinen Gehalt dar. Der Akt der symbolischen Selbstdeutung, durch den das Subjekt der Religion zugleich mit seinem Gehalt entsteht, ist dabei immer in eine konkrete Kultur und ihre kommunikativen Formen eingebunden.

Die Dogmatik konstruiert den Religionsbegriff in der Theologie. Sie ist also selbst eine Deutung der als Glaube weiterbestimmten Religion. Die dogmatischen Symbole deuten und strukturieren den Akt der symbolischen Selbstdarstellung des Selbst. Gotteslehre, Christologie und Pneumatologie haben den Status von Reflexionsebenen des Glaubens, in denen dieser Vollzug sich in unterschiedlichen Hinsichten darstellt. In dem Akt selbst - also in dem kommunikativ vermittelten Geschehen von menschlicher Selbsterschlossenheit - liegen diese Ebenen zusammen vor und überlagern sich. ${ }^{16}$ In der dogmatischen Gotteslehre stellt sich der religiöse Akt im Hinblick auf seine Unableitbarkeit und Vollzugsgebundenheit dar. Gott ist allein im Vollzug des Glaubens beim Menschen. ${ }^{17}$ Die Transzendenz und Unbedingtheit Gottes repräsentiert das Geschehen von menschlicher Selbsterschlossenheit sowie deren Bindung an ihren Vollzug.

Religion als Vollzug und Darstellung menschlichen SichVerstehens entsteht unableitbar in der Geschichte. Die symbolischen Bilder, mit denen sich ein Selbst beschreibt und herstellt, verdanken sich durchweg einer inhaltlich konkreten Geschichte. Ein Selbst existiert allein in und als Produktion seiner symbolischen Selbstdarstellung. Anders als in einem konkreten Selbstverständnis kann es sich weder erfassen noch verständlich werden. Es ist jedoch nie mit dem Bild, welches es von sich hat, identisch. Der Zusammenhang von Selbsterschlossenheit und deren symbolischer Darstellung ist der Gegenstand der christologischen Weiterbestimmung des religiösen Aktes. Auch die Christologie ist kein inhaltlicher Bestandteil des Glaubens, sondern dessen Reflexionsebene. Der Glaube stellt sich im Christusbild selbst als ein reflexiver Akt dar.

Mit der theologischen und christologischen Beschreibung des religiösen Aktes ist dieser nun noch nicht vollständig

15.Vergleiche Nassehi (2008:173): 'Denn das die Sinnform des Religiösen in de Gesellschaft wie alle anderen sozialen Sinnformen als Kommunikation vorkommt, die in erster Linie von ihrer Anschlussfähigkeit abhängig ist, verweist darauf, dass sich das Religiöse als Personenmerkmal nur sehr unzureichend bestimmen lässt weder als Personenmerkmal in Forschungsdesigns, die solche abfragen, noch als ein in (1997:163-174).

16.Das hat auch die alte Dogmatik mit ihrem Grundsatz, die Werke der Trinität nach außen seien ungeteilt, stets betont.

17.In diesem Sinne ist mit Eberhard Jüngel zu formulieren, Gottes Sein ist im Werden. Vergleiche Jüngel (1986). expliziert. Jede religiöse Kommunikation und jede menschliche Selbst- und Weltdeutung, so sehr sie auch nur durch eine Herstellung von symbolischen Formen möglich ist, ist an eine konkrete, inhaltliche Kultur gebunden. Der Deutungsakt, durch den ein religiöses Selbst erst entsteht, ist selbst schon konkret bestimmt. Die Einbindung dieses Aktes in eine inhaltliche religiöse Tradition sowie deren transformierende Aneignung ist das Thema Pneumatologie.

\section{Die systematische Funktion des Heiligen Geistes}

Die Reformatoren hatten das Wirken des Heiligen Geistes an den Buchstaben der Schrift gebunden und die soteriologische Dimension der Pneumatologie betont. Die heilschaffende innere Wirksamkeit des Geistes ist an den äußeren Buchstaben als Vehikel gebunden. Im Gottesdienst verkündigen die Prediger das Wort Gottes. Sie treiben es in die Ohren, aber der Geist treibt es ins Herz eines Menschen. Die historische Kritik der Neuzeit hat das reformatorische Schriftverständnis aufgelöst. Unter ihren Bedingungen kann die Bibel nicht mehr als ein in sich klares und selbstsuffizientes Buch verstanden werden, welches gleichsam wortwörtlich von Gott eingegeben ist. Sie wird selbst $\mathrm{zu}$ einem religionsgeschichtlichen Dokument, in dem sich die Geschichte der israelitischen Religion sowie die des frühen Christentums niedergeschlagen hat. Der genannte Umbruch bleibt nicht ohne Folgen für die reformatorische Zuordnung von Geist und Buchstabe. Ersterer kann nicht mehr an eine quasi objektive Wahrheitsinstanz gebunden sein, die, aufgrund ihrer göttlichen Herkunft, zeitlos gültig ist. Die reformatorische Bindung des Geistes an den Buchstaben repräsentiert, so muss man unter den veränderten Bedingungen der Moderne den Gedanken umformulieren, die inhaltlich-geschichtliche Einbindung jedes Selbstdeutungsaktes. In dieser Form ist der Geist jedoch für die Beschreibung der Religion auch notwendig.

Glaube ist das Geschehen von menschlicher Selbsterschlossenheit und deren Darstellung. Darin liegt beschlossen, dass Religion an diesen Vollzug gebunden ist und erst in und mit ihm entsteht. Der Deutungsakt, in dem das religiöse Subjekt zusammen mit seinem Gehalt zustande kommt, ist in eine inhaltlich bestimmte und geschichtlich gewordene Kultur eingebunden. Jede Überlieferung, von der Sprache bis hin $\mathrm{zu}$ den kulturellen und religiösen Zeichensystemen, muss von dem Einzelnen empfangen und angeeignet werden. Kommunikation, auch die religiöse, verwirklicht sich ausschließlich in der Spannung von geschichtlicher Abhängigkeit und Transformation der überlieferten Gehalte im Akt von deren deutender Aneignung. ${ }^{18}$ Die Einbindung des religiösen Vollzugs in die christlich-protestantische Tradition und deren institutionelle Vermittlung zu reflektieren, ist die systematische Funktion der Pneumatologie. Die reformatorische Auffassung von dem an das Wort der Schrift gebundenen Geist ist also in einer deutungs- und symboltheoretischen Religionstheorie

18.Diesen Aspekt hatte bereits Ernst Troeltsch in den Fokus seiner Neubestimmung der Pneumatologie gerückt. Vergleiche Troeltsch (1925:345-347). Vergleiche hierzu Wittekind (2014:44f.). 
aufzunehmen und weiterzuführen. Religion entsteht beim Einzelnen allein in kommunikativen Prozessen.

Mit dem Symbol 'Heiliger Geist' deutet sich der religiöse Akt selbst als ein Geschehen in der Geschichte. Der religiöse Vollzug stellt mit dem Gottesgeist seine Selbsterschlossenheit in ihrer notwendigen Einbindung in eine konkrete Tradition dar. Das Thema der Pneumatologie ist folglich die Traditionsgebundenheit von religiösen Selbst- und Weltdeutungen, deren Kontingenz und Wandelbarkeit. Die Lehre vom Geist ist als dogmatische Reflexion der notwendigen inhaltlichen Bestimmtheit der Religion auszuarbeiten. Religiöse Selbstdeutungen sind durchweg von Überlieferungen abhängig, die christlichen von der Bibel und ihrer Traditionsvermittlung. Die symbolischen Formen, in denen sich religiöse Gewissheiten darstellen, sind geschichtlich geworden. Sie könnten damit grundsätzlich anders sein, als sie sind. Allerdings müssen sie auch als andere notwendig konkret sein. Indem die Pneumatologie die notwendige inhaltliche Bestimmtheit des religiösen Deutungsaktes thematisiert, reflektiert sie deren geschichtliche Kontingenz und Wandelbarkeit.

Sowohl die neutestamentlichen Texte als auch die theologische Lehrtradition haben den Heiligen Geist als Gabe verstanden. Er erinnert an Christus. Darin besteht sein heiligendes Werk. In der Pneumatologie wird die Abhängigkeit religiöser Selbstdeutungen von geschichtlich vermittelten Traditionen zum Thema der theologischen Beschreibung von Religion. Es geht also in der Lehre vom Geist nicht um dessen universale Identifizierung in der Welt. Wird das Wirken des Geistes in der Welt der Religionen oder in den vielfältigen Befreiungs- oder Aufbruchserfahrungen postuliert, dann wird die Pneumatologie nicht nur diffus, es gibt auch kein Kriterium zur Scheidung der Geister mehr. In den Fokus der Geistlehre hat deshalb die Bestimmung der Identität der christlichen Religion in der Geschichte zu rücken. Das war indes bereits das Anliegen der Schriftlehre. Deren systematischer Ort im Aufbau der theologischen Dogmatik ist demzufolge die Pneumatologie und nicht - wie in der alten Dogmatik - die Prolegomena. ${ }^{19}$ Die Schrift repräsentiert die notwendige inhaltliche Bestimmtheit des religiösen Selbstdeutungsaktes. Das allein ist der Sinn des extra nos der Schrift. In dem religiösen Aneignungsakt werden die überlieferten Gehalte zugleich rezipiert und transformiert. Als bewusster Vollzug realisiert sich Religion ausschließlich in der Spannung von Abhängigkeit von bestimmten Gehalten und deren produktiver Umformung. Das ist aber auch allein die Weise, in der sich die Identität der christlichen Religion in der Geschichte verwirklicht. Die systematische Funktion der Schriftlehre

19. Bereits Schleiermacher hatte in seiner Glaubenslehre vor dem Hintergrund der Auflösung des protestantischen Schriftprinzips dafür plädiert, die Schriftlehre in der Pneumatlogie auszuarbeiten. Vergleiche Schleiermacher (1999:II/284): ' $\$ 128$. Das Ansehen der Heiligen Schrift kann nicht den Glauben an Christus begründen, vielmehr muß dieser schon vorausgesetzt werden, um der Heiligen Schrift ein besonderes Ansehen einzuräumen.' Die protestantische Theologie des 20 Jahrhunderts ist ihm darin weitgehend gefolgt, auch wenn sie aus didaktischen Grunden die Schriftlehre in den Prolegomena verhandelt hat. Vergleiche Kaftan (1897:44-56); Althaus (1933.31): 'Alles was uber die Merkmale gottlicher Autorität gesagt werden kann, setzt die Anerkennung der Schrift schon voraus und bedeute also ein Gehen auf der inneren Linie des Glaubens.' besteht somit darin, die geschichtliche Bestimmtheit der religiösen Selbstdeutungen durch inhaltliche Gehalte sowie die Notwendigkeit ihrer individuellen Aneignung und Umformung als Darstellungen religiöser Gewissheit zum Thema der theologischen Reflexion zu machen.

Eine Identität der christlichen Religion gibt es lediglich als wandelbare Selbstbeschreibungen und Selbstdarstellungen. Deshalb sind ihre Grenzen schon innerhalb des Christentums notorisch umstritten. Unter den Bedingungen des modernen Pluralismus sowie der grundgesetzlich garantierten Religionsfreiheit in demokratischen Gesellschaften haben sich die christlich-religiösen Selbstbeschreibungen pluralisiert. Das moderne Christentum kommuniziert sein Selbstverständnis schon längst nicht mehr ausschließlich mit biblischen Bildern, auch wenn ihnen noch ein grundlegender Stellenwert zukommt. Überlieferte christliche Symbole - wie Gott, Christus und der Heilige Geist - werden angereichert mit höchst unterschiedlichen Vorstellungsgehalten, welche die Religionskultur der Moderne bereitstellt. Ohne solche 'Amalgamierungen' und Synkretismen kann das Christentum als geschichtliche Religion gar nicht existieren. Aber wie viel Umformung verträgt eine Religion, um noch dieselbe $\mathrm{zu}$ sein? Bei welchem Intensitätsgrad der Inkulturation hört das Christentum auf, christlich zu sein?

Die Pneumatologie reflektiert die Grenzen der Transformation der christlichen Selbstdeutungen. Das Kriterium ihrer Grenzreflexion kann nach der Auflösung des Schriftprinzips des alten Protestantismus sowie der Einsicht in die für den religiösen Akt konstitutive Spannung von inhaltlicher Abhängigkeit und deren produktiver Umformung nicht mehr inhaltlich beschrieben werden. Die Kriterienfunktion der inhaltlichen Bestandteile des Glaubensbegriffs ist durch die Selbstdurchsichtigkeit des religiösen Vollzugs zu ersetzen. Die Selbsterschlossenheit des Menschen als endliche Freiheit bildet das Kriterium der Umformung der überlieferten christlichen Gehalte. Die alte Dogmatik hatte nicht ohne Grund im Anschluss an Paulus den Heiligen Geist an Christus gebunden. Der Geist, der in die Wahrheit führt, ist der Geist Christi. Anders als in der religiösen Kommunikation ist Christus jedoch nicht gegenwärtig. Nur auf diese Weise kann er von dem Einzelnen als Bild des Glaubens von sich selbst als Gottesverhältnis angeeignet werden.

In der dogmatischen Pneumatologie wird somit die Identität des geschichtlichen Christentums zum Thema der theologischen Reflexion. Darin besteht ihr eigenes Thema. Es ist für eine angemessene Erfassung und Explikation der reflexiven Struktur des religiösen Aktes unverzichtbar. ${ }^{20}$ Begreift man darin die Aufgabe der Geistlehre, dann besteht ihre systematische Funktion in einer Selbstbeschreibung der christlichen Religion als Aneignung von wandelbaren symbolischen Formen. Das unterscheidet die Lehre vom Heiligen Geist sowohl von der Gotteslehre als auch von der Christologie.

20.Vergleiche die Kritik von Stoellger (2014:464-493), an deutungstheoretischen Religionsbegriffen, die darauf zielt, dass der Deutungsakt stets schon ein bestimmter ist. 


\section{Acknowledgements Competing interests}

The authors declare that they have no financial or personal relationships which may have inappropriately influenced them in writing this article.

\section{References}

Althaus, P., 1933, Grundriß der Dogmatik. 1. Teil, 4. Aufl. Verlag der Universitätsbuchhandlung Rudolf Merkel, Erlangen.

Barth, K., 1930, 'Der heilige Geist und das christliche Leben', in H. Barth (Hrsg.), Zur Lehre vom heiligen Geist, pp. 39-105, Christian Kaiser, München.

Barth, U., 2003, Religion in der Moderne, Mohr Siebeck, Tübingen.

Bernhardt, R., 2000, 'Trinitätstheologie als Matrix einer Theologie der Religionen', in Ökumenische Rundschau 49, 287-301.

Bernhardt, R., 2005a, Ende des Dialogs? Die Begegnung der Religionen und ihre theologische Reflexion, Theologischer Verlag Zürich, Zürich.

Bernhardt, R., 2005b, 'Protestantische Religionstheologie auf trinitätstheologischem Grund', in C. Danz \& U.H.J. Körtner (Hrsg.), Theologie der Religionen. Positionen und Perspektiven evangelischer Theologie, pp. 107-120, Neukirchener Verlag, Neukirchen-Vluyn.

Danz, C., 2015a, 'Der Geist der Religion. Anmerkungen zur religionstheoretischen Funktion der Pneumatologie', in Erleben und Deuten. Dogmatische Reflexionen
im Anschluss an Ulrich Barth. Festschrift zum 70. Geburtstag, R. Barth, A. Kubik \& im Anschluss an Ulrich Barth. Festschrift zum 70. Geburtstag,
A. v. Scheliha (Hrsg.), pp. 257-272, Mohr Siebeck, Tübingen.

Danz, C., 2015b, 'Hominem iustificari fide. Überlegungen zur protestantischen Anthropologie', in B. Stubenrauch \& M. Seewald (Hrsg.), Das Menschenbild der Anthropologie, in B. Stubenrauch \& M. Seewald (Hrsg.), Das Menschenbild der
Konfessionen-Achillesferse der Ökumene?, pp. 157-185, Verlag Herder, Freiburg i. Br.

Danz, C., 2008, Die Deutung der Religion in der Kultur. Aufgaben und Probleme der Theologie im Zeitalter des religiösen Pluralismus, Neukirchener Verlag, Neukirchen-Vluyn.

Danz, C., 2010, Einführung in die evangelische Dogmatik, Wissenschaftliche Buchgesellschaft, Darmstadt.

Danz, C., 2013, Einführung in die Theologie Martin Luthers, Wissenschaftliche Buchgesellschaft, Darmstadt.

Danz, C. \& Murrmann-Kahl, M. (Hrsg.), 2014, Zwischen Geistvergessenheit und Geistversessenheit. Perspektiven der Pneumatologie im 21. Jahrhundert, Moh Siebeck, Tübingen

Deuser, H., 1999, Kleine Einführung in die Systematische Theologie, Reclam, Stuttgart.

Düsing, E., Neuer, W. \& Klein, H-D. (Hrsg.), 2009, Geist und Heiliger Geist. Philosophische und theologische Modelle von Paulus und Johannes bis Barth und Balthasar Königshausen \& Neumann, Würzburg.

Henning, C., 2000, Die evangelische Lehre vom Heiligen Geist und seiner Person. Studien zur Architektur protestantischer Pneumatologie im 20. Jahrhundert Gütersloher Verlagshaus, Gütersloh.

Hirsch, E., 1937, Hilfsbuch zum Studium der Dogmatik. Die Dogmatik der Reformatoren und der altevangelischen Lehrer quellenmäßig belegt und verdeutscht, Walter de Gruyter, Berlin.
Jüngel, E., 1986, Gottes Sein ist im Werden. Verantwortliche Rede vom Sein Gottes bei Karl Barth. Eine Paraphrase, 4. Aufl., Mohr Siebeck, Tübingen.

Kaftan, J., 1897, Dogmatik, Mohr Siebeck, Freiburg.

Kähler, M., [1905] 1994, Die Wissenschaft von der christlichen Lehre von dem evangelischen Grundartikel aus im Abrisse dargestellt, 3. Aufl., Leipzig, ND Spenner, Waltrop.

Luhmann, N., 1997, 'Vom Sinn religiöser Kommunikation', in K. Gabriel, A. Herlth \& K.P. Strohmeier (Hrsg.), Modernität und Solidarität. Konsequenzen gesellschaftlicher Modernisierung. Für Franz-Xaver Kaufmann, pp.163-174, Verlag Herder, Freiburg.

Luther, M., 1982, Der kleine Katechismus, in Die Bekenntnisschriften der evangelischlutherischen Kirche, 9. Aufl., pp. 499-542, Vandenhoeck \& Ruprecht, Göttingen.

Moltmann, J., 1968, 'Die Wahrnehmung der Geschichte in der christlichen Sozialethik', in Bertelsmann Stiftung (Hrsg.), Perspektiven der Theologie. Gesammelte Aufsätze, pp. 149-173, Christian Kaiser, München.

Moltmann, J., 1991, Der Geist des Lebens. Eine ganzheitliche Pneumatologie, Christian Kaiser, München.

Nassehi, A., 2008, 'Religiöse Kommunikation: Religionssoziologische Konsequenzen einer qualitativen Untersuchung', in Bertelsmann Stiftung (Hrsg.) Woran glaubt die Welt? Analysen und Kommentare zum Religionsmonitor 2008, pp. 169-203, Bertelsmann Stiftung, Gütersloh.

Riesebrodt, M., 2007, Cultus und Heilsversprechen. Eine Theorie der Religion, C.H. Beck, München.

Ritschl, A., 1890, Unterricht in der christlichen Religion, 4. Aufl., Adolph Marcus, Bonn.

Ritschl, D., 1993, s.v. 'Pneumatologie. 2. Systematisch-theologisch', in E. Fahlbusch (Hrsg.), Evangelisches Kirchenlexikon, Bd. 3, Vandenhoeck \& Ruprecht, Göttingen, pp. 1247-1252.

Ritschl, A., 1999, 'Die christliche Vollkommenheit. Ein Vortrag', in F. Hofmann (Hrsg.), Kleine Schriften, pp. 44-64, Spenner, Waltrop.

Schleiermacher, F., [1830-1831], 1999, Der christliche Glaube, M. Redeker (Hrsg.) Walter de Gruyter, Berlin.

Stoellger, P., 2014, 'Theologie als Deutungsmachttheorie. Zur Hermeneutik von Deutungsmacht im systematischen Diskurs', in P. Stoellger (Hrsg.), Deutungsmacht. Religion und belief systems in Deutungsmachtkonflikten, pp. 431-523, Mohr Siebeck, Tübingen.

Tillich, P., 1966, Systematische Theologie, Bd. 3, Evangelisches Verlagswerk, Stuttgart. Trillhaas, W., 1962, Dogmatik, Walter de Gruyter, Berlin.

Troeltsch, E., 1925, Glaubenslehre. Nach Heidelberger Vorlesungen aus den Jahren 1911 und 1912, G. von le Fort (Hrsg.), Duncker \& Humblot, München.

Wagner, F., 1991, Was ist Religion? Studien zu ihrem Begriff und Thema in der Geschichte und Gegenwart, 2. Aufl., Gütersloher Verlagshaus, Gütersloh.

Welker, M., [1992] 2013, Gottes Geist. Theologie des Heiligen Geistes, 5. Aufl., Neukirchener Verlag, Neukirchen-Vluyn.

Wittekind, F., 2008, 'Zwischen Religion und Gott. Überlegungen zum Selbstverständnis und zur Begründung einer protestantischen dogmatischen Theologie', in H. NaglDocekal \& F. Wolfram (Hrsg.), Jenseits der Säkularisierung. Religionsphilosophische Studien, pp. 351-384, Parerga, Berlin. Wittekind, F., 2014, 'Theologiegeschichtliche Überlegungen zur Pneumatologie', in C. Danz \& M. Murrmann-Kahl (Hrsg.), Zwischen Geistvergessenheit und Geistversessenheit. Perspektiven der Pneumatologie im 21. Jahrhundert, pp. 13-67, Mohr Siebeck, Tübingen. 